\title{
The prognostic significance of preexisting diabetes in patients with surgically treated renal cell carcinoma: the ongoing debate
}

\author{
Ho Won Kang, Yong-June Kim \\ Department of Urology, Chungbuk National University Hospital, Chungbuk National University College of Medicine, Cheongju, South Korea \\ Correspondence to: Yong-June Kim, MD, PhD. Department of Urology, Chungbuk National University College of Medicine, 1st Chungdae-ro, \\ Seowon-gu, Cheongju, Chungbuk 28644, South Korea. Email: urokyj@cbnu.ac.kr. \\ Provenance: This is an invited article commissioned by the Section Editor Dr. Xiao Li (Department of Urology, Jiangsu Cancer Hospital, Jiangsu \\ Institute of Cancer Research, Nanjing Medical University Affiliated Cancer Hospital, Nanjing, China). \\ Comment on: Nayan M, Jalali S, Kapoor A, et al. Diabetes and kidney cancer survival in patients undergoing nephrectomy: A Canadian multi-center, \\ propensity score analysis. Urol Oncol 2019;37:576.e11-6.
}

Submitted Sep 13, 2019. Accepted for publication Sep 26, 2019.

doi: 10.21037/atm.2019.09.131

View this article at: http://dx.doi.org/10.21037/atm.2019.09.131

Because renal cell carcinoma (RCC) patients are likely to have substantial comorbidities due to advanced age at the time of diagnosis, it is important to explore the effects of preexisting medical diseases on survival after definitive surgical treatment (1). Several epidemiological studies have detected a significant association between RCC prognosis and metabolic syndrome components, such as obesity, hypertension, diabetes mellitus (DM), and dyslipidemia (2-5). Although obesity is a well-known risk factors for RCC development, growing evidence suggests that high body mass index (BMI) is significantly associated with favorable overall survival (OS), cancer-specific survival (CSS), and recurrence-free survival (RFS) in patients with RCC, paradoxically (3). The relationship between hypertension and the risk of RCC remains still controversial, whereas evidence suggests that tyrosine kinase inhibitor-associated hypertension may be a correlative biomarker of antitumor efficacy in metastatic RCC $(4,6)$. An adverse correlation between preexisting DM and tumor recurrence or survival has been reported for several malignancies such as liver, breast, colorectal and endometrial cancers (7-10). With regard to RCC, some studies suggest that DM is associated significantly with a poor prognosis after nephrectomy, while others show no significant association (5,11-14). This controversy might stem from study limitations such as relatively small sample sizes and short follow-up times, the absence of data about potential confounders, inclusion of heterogeneous RCC histological subtypes. Long-time interval also warrant consideration which could represent a confounding factor for the analysis.

In a recent article by Nayan et al. in 'Urologic Oncology: Seminars and Original Investigations', the authors conducted a multi-center study to determine whether preexisting $\mathrm{DM}$ can predict the prognosis of clinically nonmetastatic RCC using the Canadian Kidney Cancer information system database (15). The investigators used propensity score methods based on various clinical, operative, and pathological characteristics to further reduce selection bias. The authors of this interesting work conclude that diabetics have similar survival outcomes compared to nondiabetics after nephrectomy. Although this multicenter study is well designed and provides more definitive information about the prognostic impact of DM on RCC survival, aforementioned long-time interval between 1989 and 2017 is one of the major drawbacks of the study. Disease management in terms of minimal invasive and nephron sparing approaches for RCC has widely evolved within the last decades and should be taken into account. Moreover, the treatment of recurrent or metastatic RCC after initial curative surgery was revolutionized with the advent of antiangiogenic drugs, tyrosine-kinase inhibitors or immune checkpoint inhibitors during the last decade. Not all patients have access to modern treatment for recurrent or metastatic RCC, which is another confounding factor given the recruitment period of the study. Conceding to this view of the subject, the investigators performed a subgroup analysis based on cohort era and demonstrated similar results to the primary analyses. On the other hand, 
contemporary series in the literature have detected a significant association between preexisting DM and cancer survival in patients with RCC. Ha et al. (11) identified diabetes as an independent prognostic factor in terms of RFS, CSS, and OS using multicenter data for 2,597 Korean patients with pT1-2 localized clear cell RCC. Another study by Lee and colleagues applied similar study design and methodology with current study. The authors conducted a propensity score matching in a 1:2 ratio for patients with diabetes and those without diabetes, respectively (12). In matched cohorts, diabetes was an independent predictor of progression free survival (HR 1.766), OS (HR 1.825) and CSS (HR 2.266). Notably, high preoperative HbA1c independently associated with progression free survival (HR 2.221) among patients with diabetes (12). Recently, Chen et al. (5) performed a meta-analysis of literature published between 2005 and 2014, which includes eighteen cohort studies. The results demonstrated that DM is significantly associated with poor OS (HR 1.56), CSS (HR 2.03), and RFS (HR 1.73) in RCC patients. In addition, DM was significantly associated with both poor OS and CSS in subgroup with localized RCC, clear cell RCC, and RCC with surgical treatment (5). Comprehensively, the question still remains whether diabetes exerting a detrimental effect on long-term survival, or whether these negative oncologic outcomes are secondary to diabetes itself in RCC. Additional well-conducted prospective studies are inevitable to demonstrate a more convincing association between DM and RCC prognosis.

In conclusion, the findings from Nayan et al. attempted to address the question of whether diabetes is associated with survival in RCC. Although current interesting study provides a highly generalizable insight into the effect of diabetes on survival, the prognostic significance of preexisting DM in RCC remains to be clarified.

\section{Acknowledgments}

None.

\section{Footnote}

Conflicts of Interest: The authors have no conflicts of interest to declare.

Ethical Statement: The authors are accountable for all aspects of the work in ensuring that questions related to the accuracy or integrity of any part of the work are appropriately investigated and resolved.

\section{References}

1. Verhoest G, Veillard D, Guille F, et al. Relationship between age at diagnosis and clinicopathologic features of renal cell carcinoma. Eur Urol 2007;51:1298-304; discussion 304-5.

2. de Martino M, Leitner CV, Seemann C, et al. Preoperative serum cholesterol is an independent prognostic factor for patients with renal cell carcinoma (RCC). BJU Int 2015;115:397-404.

3. Choi Y, Park B, Jeong BC, et al. Body mass index and survival in patients with renal cell carcinoma: a clinical-based cohort and meta-analysis. Int J Cancer 2013;132:625-34.

4. Bono P, Elfving H, Utriainen T, et al. Hypertension and clinical benefit of bevacizumab in the treatment of advanced renal cell carcinoma. Ann Oncol 2009;20:393-4.

5. Chen L, Li H, Gu L, et al. The Impact of Diabetes Mellitus on Renal Cell Carcinoma Prognosis: A MetaAnalysis of Cohort Studies. Medicine (Baltimore) 2015;94:e1055.

6. Kang HW, Seo SP, Kim WT, et al. Low preoperative serum cholesterol level is associated with aggressive pathologic features and poor cancer-specific survival in patients with surgically treated renal cell carcinoma. Int J Clin Oncol 2018;23:142-50.

7. Peairs KS, Barone BB, Snyder CF, et al. Diabetes mellitus and breast cancer outcomes: a systematic review and metaanalysis. J Clin Oncol 2011;29:40-6.

8. Barone BB, Yeh HC, Snyder CF, et al. Long-term allcause mortality in cancer patients with preexisting diabetes mellitus: a systematic review and meta-analysis. JAMA 2008;300:2754-64.

9. Mills KT, Bellows CF, Hoffman AE, et al. Diabetes mellitus and colorectal cancer prognosis: a meta-analysis. Dis Colon Rectum 2013;56:1304-19.

10. Yang WS, Va P, Bray F, et al. The role of pre-existing diabetes mellitus on hepatocellular carcinoma occurrence and prognosis: a meta-analysis of prospective cohort studies. PLoS One 2011;6:e27326.

11. Ha YS, Kim WT, Yun SJ, et al. Multi-institutional analysis of localized renal cell carcinoma that demonstrates the impact of diabetic status on prognosis after nephrectomy. Ann Surg Oncol 2013;20:3662-8.

12. Lee H, Kwak C, Kim HH, et al. Diabetes Mellitus as an Independent Predictor of Survival of Patients Surgically 
Treated for Renal Cell Carcinoma: A Propensity Score Matching Study. J Urol 2015;194:1554-60.

13. Höfner T, Zeier M, Hatiboglu G, et al. The impact of type 2 diabetes on the outcome of localized renal cell carcinoma. World J Urol 2014;32:1537-42.

14. Nayan M, Finelli A, Jewett MA, et al. Diabetes and kidney cancer outcomes: a propensity score analysis. Endocrine 2017;55:470-7.

15. Nayan M, Jalali S, Kapoor A, et al. Diabetes and kidney cancer survival in patients undergoing nephrectomy: A Canadian multi-center, propensity score analysis. Urol Oncol 2019;37:576.e11-6.
Cite this article as: Kang HW, Kim YJ. The prognostic significance of preexisting diabetes in patients with surgically treated renal cell carcinoma: the ongoing debate. Ann Transl Med 2019;7(Suppl 8):S321. doi: 10.21037/atm.2019.09.131 